\title{
When is high-dose intravenous iron repletion needed? Assessing new treatment options
}

\author{
This article was published in the following Dove Press journal: \\ Drug Design, Development and Therapy \\ 19 January 2011 \\ Number of times this article has been viewed
}

\section{David Gozzard}

Betsi Cadwaladr University Health Board, Llandudno, Wales, UK
Correspondence: David Gozzard Betsi Cadwaladr University Health Board, Llandudno, Wales, UK

Tel 01745534391

Fax 01745 583।43

Email david.gozzard@wales.nhs.uk
Abstract: High doses of intravenous iron have a role in the treatment of a number of clinical situations associated with iron deficiency, iron deficiency anemia, and blood loss. In the presence of functioning erythropoiesis, iron supplementation alone may be adequate to replenish iron stores and restore blood loss. Where hormone replacement with an erythropoiesis-stimulating agent is required, iron adequacy will optimize treatment. Intravenous iron offers a rapid means of iron repletion and is superior to oral iron in many circumstances, especially in the presence of anemia of chronic disease, where it appears to overcome the block to absorption of iron from the gastrointestinal tract and immobilization of stored iron. The clinical situations where high doses of iron are commonly required are reviewed. These include nondialysis-dependent chronic kidney disease, inflammatory bowel disease, obstetrics, menorrhagia, and anemia associated with cancer and its treatment. The literature indicates that high doses of iron are required, with levels of $1500 \mathrm{mg}$ in nondialysis-dependent chronic kidney disease and up to $3600 \mathrm{mg}$ in inflammatory bowel disease. New formulations of intravenous iron have recently been introduced that allow clinicians to administer high doses of iron in a single administration. Ferumoxytol is available in the US, has a maximum dose of $510 \mathrm{mg}$ iron in a single administration, but is limited to use in chronic kidney disease. Ferric carboxymaltose can be rapidly administered in doses of $15 \mathrm{mg} / \mathrm{kg}$ body weight, up to a ceiling dose of $1000 \mathrm{mg}$. A test dose is not required, and it can be used more widely across a spectrum of iron deficiency and iron deficiency anemia indications. The latest introduction is iron isomaltoside 1000. Again, a test dose is not required, and it can be delivered rapidly as an infusion (in an hour), allowing even higher doses of iron to be administered in a single infusion, ie, $20 \mathrm{mg} / \mathrm{kg}$ body weight with no ceiling. This will allow clinicians to achieve high-dose repletion more frequently as a single administration. Treatment options for iron repletion have taken a major leap forward in the past two years, especially to meet the demand for high doses given as a single administration.

Keywords: iron isomaltoside 1000, intravenous iron, iron deficiency anemia, iron deficiency, high dose, single dose, erythropoiesis

\section{Introduction}

Iron has an essential role in human physiology, albeit the total amount in a healthy individual is only approximately 3 grams. The balance of iron in the body broadly reflects three dynamics, ie, intake, losses, and metabolic demands. Intake will relate to the iron content in food, the volume ingested, and the ability to absorb iron from the digestive tract. This may be impaired due to pathology in the gastrointestinal tract or comorbidity (eg, a chronic inflammatory condition) resulting in expression of the regulatory peptide, hepcidin, which will block iron absorption. ${ }^{1}$ Loss may be 
a result of acute trauma or through continual chronic blood loss from the gastrointestinal, urinary, or respiratory tract, or be associated with renal replacement (hemodialysis) in chronic kidney disease. ${ }^{2}$ Increased demand occurs in infancy and childhood, pregnancy, and during use of an erythropoiesis-stimulating agent (ESA). ${ }^{3}$ Stimulation of erythropoiesis using an ESA can result in functional iron deficiency where the demand for iron increases but cannot be met from existing iron stores. ${ }^{4}$ This may be because of low iron store levels (absolute iron deficiency) or limited by iron release from storage depots. ${ }^{4}$

Historically, the oral route of administration was given much attention, but the effectiveness of oral formulations is compromised by poor absorption, poor compliance, side effects (up to $56 \%$ ), and discontinuation of treatment (19\%). ${ }^{5}$

The challenge of treating iron deficiency is related to the toxicity of iron in its elemental state, the required dose, and desired rate of repletion. Early parenteral formulations, now withdrawn in many countries, were associated with untoward effects that limited their use. ${ }^{6}$ These were surpassed with the introduction of iron sucrose and modified formulations of iron dextran (and ferric gluconate complex in some countries). ${ }^{7-9}$ These formulations had much improved safety profiles and lower rates of adverse events. The next inevitable challenge was to address the issue of eliminating the need for a test dose and being able to administer larger amounts of iron in a shorter period of time, thereby potentially improving the convenience of treatment.

Three new intravenous iron preparations have recently been introduced offering higher single-dose options. None of these preparations require a test dose, and all can be delivered rapidly. Ferumoxytol (AMAG Pharmaceuticals Inc, Cambridge, MA) is approved for the sole indication of adult iron deficiency anemia associated with chronic kidney disease. Up to $510 \mathrm{mg}$ iron can be delivered in a single dose. ${ }^{10}$ Ferric carboxymaltose (Vifor Pharma, Switzerland) has been introduced for intravenous iron supplementation, allowing doses of $15 \mathrm{mg} / \mathrm{kg}$ body weight (up to a maximum dose of $1000 \mathrm{mg}$ ) to be delivered in a single administration. ${ }^{11}$ Subsequently, iron isomaltoside 1000 (Pharmacosmos, Holbaek, Denmark) has been approved for marketing in Europe, and allows higher maximum doses in a single infusion at up to $20 \mathrm{mg} / \mathrm{kg}$ body weight. ${ }^{12}$

This review highlights the clinical situations where the requirements for high dose iron repletion are particularly relevant, and considers the practicalities associated with treatment. The focus is on iron isomaltoside 1000 and ferric carboxymaltose because the use of ferumoxytol is limited to patients with chronic kidney disease and geographically is available only in the US.

\section{Methods}

Literature (database) searches were undertaken and focused on guidelines for the diagnosis and treatment of iron deficiency, iron deficiency anemia, and anemia of chronic disease to identify the indications for which intravenous iron supplementation is a suggested therapy. Searches were undertaken to identify clinical studies investigating the role of intravenous iron in treating iron deficiency, iron deficiency anemia, and anemia of chronic disease to provide further insights into these conditions and the clinical circumstances in which high-dose intravenous iron administration would be appropriate. This search included papers reporting studies undertaken with the new preparations. The dosing parameters and product specifications from the manufacturers' summaries of product characteristics were inspected so that comment could be made on the appropriateness of the two formulations for use across a range of indications. Epidemiological survey data were used to establish mean population weights for adults by gender for Europe and the US.

\section{Results}

Firstly, it is important to consider the implications of delivering iron as a single high-dose intravenous iron supplement as opposed to more frequent small-dose administrations.

Infrequent high-dose administrations of intravenous iron reduce the frequency of hospital or clinic visits, thereby causing less disruption to patients' lifestyle, especially if working. ${ }^{13,14}$ The overall time spent attending a hospital or clinic can be reduced. This is likely to be more convenient for both patients and health care professionals and to improve patient compliance. ${ }^{14}$ Such high-dose administrations have been shown to reduce waiting lists (by increasing patient throughput). ${ }^{13}$ Travel costs are reduced, which may benefit patients, the health care economy, and the insurance company. ${ }^{13}$

From a practical clinical perspective, target hemoglobin and ferritin levels may be achieved more rapidly, allowing hematinic levels to achieve stability earlier than when using multiple small doses, which can be assessed when taking anemia management decisions. ${ }^{13}$ Reduced frequency of venous access should reduce the risk of infection and preserve veins for future access. A further major potential clinical benefit is that high doses of intravenous iron appear to overcome the block caused by hepcidin in patients with anemia of chronic disease. ${ }^{1,15}$ This may be highly significant, allowing treatment of anemia in patients with inflammatory conditions. 


\section{Nondialysis-dependent phase of chronic kidney disease}

The treatment of anemia associated with chronic kidney disease has been transformed since ESAs were introduced. Prior to the availability of ESAs, the administration of blood transfusions to address anemia associated with chronic kidney disease was commonplace. This practice was inconvenient, costly (albeit blood costs are "lost" in some health economies), and jeopardized the prospects of finding a matching organ for transplantation. ${ }^{16}$ Guidelines indicate that blood transfusions, especially for individuals in whom renal transplantation is an option, should be avoided if possible. ${ }^{17}$

Managing anemia of chronic kidney disease as the disease progresses improves the prognosis. ${ }^{18}$ In the predialysis phase (low clearance, nondialysis-dependent) hemoglobin adequacy may be sustained with iron supplementation alone, albeit most patients will ultimately progress to requiring an ESA. ${ }^{19}$

It is during this low clearance, nondialysis-dependent phase of the disease that patients benefit from high doses of intravenous iron. Using the Ganzoni formula, a suitable intravenous iron dose can be calculated. ${ }^{11,12,20}$ Infrequent high-dose intravenous iron supplementation will sustain iron adequacy, whilst avoiding the need to attend the renal department solely to receive iron supplementation, with intervals between repeated administrations of up to a year or more. ${ }^{21}$ This can help preserve lifestyle and employment as the disease progresses.

Before an ESA is commenced, iron status should be optimized to avoid functional iron deficiency. This again may be achieved by administering a single high dose of intravenous iron. The benefit of a single dose, compared with administering a number of smaller doses, is that iron metabolism stability is achieved more rapidly. The use of frequent smaller doses of intravenous iron (once or twice a week) will take days or weeks longer to administer the required dose, and then a further three weeks from the final dose to achieve stable conditions when hematinic levels can be confidently assessed, making anemia management more difficult. ${ }^{21}$ Iron requirements of 1000-1500 mg or higher are likely to be required to achieve target ferritin and hemoglobin levels. ${ }^{21}$

For patients subsequently electing to have chronic ambulatory peritoneal dialysis as their mode of renal replacement, high-dose intravenous iron supplementation is often preferred because this continues to obviate the need for frequent hospital attendance to receive intravenous iron supplementation.

\section{Inflammatory bowel disease}

Anemia occurs in an estimated one-third of patients with inflammatory bowel disease, which can have a profound impact on patient quality of life. ${ }^{22}$ Anemia associated with inflammatory bowel disease commonly reflects a mixed etiology of iron deficiency anemia and anemia of chronic disease. ${ }^{22}$ It is suggested that treatment should be considered for all patients with a hemoglobin level below normal. ${ }^{23}$ The international guidelines suggest that anemic patients will rarely have an iron deficit of less than $1000 \mathrm{mg}$ of iron. ${ }^{23}$ These guidelines suggest using transferrin saturation levels to monitor intravenous iron treatment. They suggest that iron administration may be continued until a limit of $50 \%$ transferrin saturation is observed. High doses of intravenous iron, up to $3600 \mathrm{mg}$ (administered as multiple small doses), have been administered to patients with inflammatory bowel disease but without liver damage or iron overload, which is unlikely to occur because of ongoing blood loss in these patients. ${ }^{23}$ Intestinal bleeding (visible or occult) is associated with inflammatory bowel disease, and may be considered as a consistent clinical feature. ${ }^{23}$

The international guidelines indicate that the preferred route for iron supplementation is intravenous. ${ }^{23}$ Intravenous iron is more effective, better tolerated, and improves quality of life to a greater extent than oral iron supplements. ${ }^{23}$ Clinical studies indicate a faster and prolonged response with intravenous iron. ${ }^{23}$ In addition to oral iron not being able to compensate for ongoing blood loss, studies in animal models of inflammatory bowel disease consistently show an increase in oxidative stress, disease activity, intestinal inflammation, and also the potential for the development of colorectal cancer. ${ }^{23}$ Other studies suggest that nutritional iron may be an exogenous promoter for the onset of colitis.

It should be recognized that high doses of intravenous iron can overcome the hepcidin-mediated block of iron absorption associated with anemia of chronic disease and also the blocked mobilization of iron stores. ${ }^{15}$ Success with intravenous iron alone can be achieved in $70 \%-80 \%$ of patients with anemia of chronic disease. ${ }^{23}$ The concomitant use of intravenous iron and an ESA achieves optimal hemoglobin levels in almost all patients. ${ }^{23}$

The goals of anemia therapy are to increase the hemoglobin, serum ferritin, and transferrin saturation above the lower threshold of normal, to prevent the hemoglobin level falling below normal, to avoid the need for a blood transfusion, and to relieve symptoms associated with anemia whilst improving quality of life. The hemoglobin improvement goal is $20 \mathrm{~g} / \mathrm{L}$ or becoming normal in four weeks. ${ }^{23}$

It is suggested that absolute indications for using intravenous iron include a hemoglobin level below $100 \mathrm{~g} / \mathrm{L}$, intolerance or suboptimal response to oral iron, severe intes- 
tinal disease activity, concomitant use of ESAs, or patient preference. $^{23}$

\section{Obstetrics}

During pregnancy, iron requirements increase from $1.5-2.0 \mathrm{mg} /$ day to $5-7 \mathrm{mg}$ /day. ${ }^{24}$ Without adequate stores, and with suboptimal intake, iron deficiency anemia may prevail. Guidelines suggest that low hemoglobin levels (below $85 \mathrm{~g} / \mathrm{L}$ ) increase the risk of poor fetal outcomes (low birth rate, preterm delivery). ${ }^{25}$

Serum ferritin levels should be used to investigate adequacy of iron stores which are considered low if below $30 \mu \mathrm{g} / \mathrm{L} .{ }^{25}$ Low iron stores can be readily depleted with blood loss at delivery, which may adversely impact the mother's levels of fatigue and energy and therefore ability to care for the neonate. This may result in a prolonged stay in hospital. ${ }^{26}$ If uncorrected, this may have implications for the baby if breastfed because, in the medium to long term, the baby will not receive adequate amounts of iron.

Postpartum hemorrhage is a major contributor to maternal mortality and morbidity. ${ }^{27} \mathrm{~A}$ recent analysis of trends indicates that there is a rise in incidence and severity. Cesarean section delivery is associated with higher volume blood loss, with $1 \%-3 \%$ of patients losing more than $1000 \mathrm{~mL}$. There is a concomitant increase in blood transfusions to treat postpartum hemorrhage. ${ }^{27}$

Low antenatal hemoglobin concentrations (and especially iron deficiency) are a risk factor for postpartum hemorrhage and severity of hemorrhage. ${ }^{28}$ Intravenous iron can be used in the antenatal period to correct iron deficiency anemia and hemoglobin levels prior to delivery seeking to reduce the risk of hemorrhage and need for peripartum blood transfusions. ${ }^{29}$

In the peripartum period (0-6 months), up to $13 \%$ of mothers are iron-deficient and $10 \%$ are anemic. ${ }^{30}$ Oral supplementation produces a slow and often suboptimal response. Intravenous iron is a safe alternative, reducing the need and volume of blood transfusions. ${ }^{29,31}$ Intravenous iron therapy is associated with a significant increase in iron stores and hemoglobin levels in this patient group. ${ }^{31}$

Studies undertaken to evaluate the response to parenteral iron administration during pregnancy and in the peripartum period indicate that high dose requirements (up to $1600 \mathrm{mg}$ ) may be required. ${ }^{29,32}$ The use of a single high-dose administration of iron was found to be beneficial in antenatal intravenous iron supplementation. Compliance can be an issue in patients prescribed smaller and more frequent doses of intravenous iron. Only $7 \%$ of patients commencing a regimen of multidose intravenous iron sucrose supplementation completed the prescribed course of treatment. ${ }^{14}$ The majority of patients $(61 \%)$ received fewer than five doses, having failed to return to the hospital for their infusions. This compared with $100 \%$ compliance for a high-dose infusion regimen. ${ }^{14}$

\section{Heavy uterine bleeding}

Heavy menstrual bleeding is a common cause of iron deficiency anemia in the developed world. Menorrhagia is associated with heavy cyclical menstrual bleeding, with the loss of $80 \mathrm{~mL}$ or more of blood per cycle. ${ }^{33,34}$ The loss of blood in a year would approximate one liter, equivalent to $1000-1500 \mathrm{mg}$ of iron per year. Anemia in women with menorrhagia is often untreated or inadequately treated. ${ }^{35}$ In several updates on the management of menorrhagia, the treatment of iron deficiency or iron deficiency anemia is not explicitly addressed. ${ }^{34,36-38}$

Studies indicate that women with heavy uterine bleeding have a high requirement for blood transfusions and have higher associated hospital costs and reduced quality of life. ${ }^{35}$ Iron supplementation to correct iron deficiency and iron deficiency anemia in women with menorrhagia can avoid blood transfusions in all but those with highly compromised hemoglobin levels. Oral iron is unlikely to keep pace with iron losses. ${ }^{39,40}$

In two studies, the effectiveness of intravenous iron supplementation has been compared with oral iron in women with iron deficiency anemia associated with menorrhagia and heavy uterine bleeding. ${ }^{39,40}$ In both studies, intravenous iron was more effective than oral iron, achieving significantly higher increases in hemoglobin levels and reaching target hemoglobin levels. In a study undertaken by Kim et al, the improvement in ferritin levels was highly notable. ${ }^{39}$ In another study by Van Wyck et al, patients treated with ferric carboxymaltose reported greater gains in vitality, physical function, and improvements in symptoms of fatigue. ${ }^{40}$ In this latter paper, patients received $1000 \mathrm{mg}$ of intravenous iron or more (repeated doses) and in the former multiples of $200 \mathrm{mg}$ (iron sucrose) three times a week until the total calculated repletion dose was achieved. High-dose intravenous iron supplementation appears to be highly effective in correcting iron deficiency and iron deficiency anemia in women with menorrhagia. ${ }^{40}$

\section{Anemia associated with cancer and its treatment}

An ESA plus intravenous iron can be used to address anemia caused by cancer treatment and can replace the use of blood transfusions. The European Organisation for Research and 
Treatment of Cancer (EORTC) guidelines recognize that treating anemia in cancer patients improves their quality of life and reduces the requirement for emergency blood transfusions which offer only temporary symptom relief whilst having a number of associated risks. They recommend early initiation of treatment (at a hemoglobin level of $90-110 \mathrm{~g} / \mathrm{L}$ ) with continuation to sustain hemoglobin levels $\leq 120-130 \mathrm{~g} / \mathrm{L}$ for patients showing symptomatic improvement. ${ }^{41}$ Optimal improvements in energy, activity, and quality of life occur when hemoglobin levels increase from $110 \mathrm{~g} / \mathrm{L}$ to $130 \mathrm{~g} / \mathrm{L}^{42}$

Cancer may be associated with anemia of chronic disease. ${ }^{43}$ It is postulated that cancer patients, with ironrestricted erythropoiesis, have iron in their stores which is not accessible for erythropoiesis. ${ }^{44}$ The access to stored iron may be inhibited by the upregulation of hepcidin through inactivation of ferroportin, the major carrier of iron. ${ }^{44}$ This mechanism appears to operate in other conditions associated with anemia of chronic disease.

There are concerns that intravenous iron is underused in oncology patients. Appropriate use improves the response to ESAs, reducing doses and costs, whilst improving their effectiveness in elevating hemoglobin levels and overcoming anemia of chronic disease. ${ }^{44-47}$ Bastit et al have shown that administration of intravenous iron significantly improves hemoglobin levels, hematopoietic response, and time to reach target hemoglobin levels when compared with oral iron administration, and has also shown a statistically significant reduction in administered blood transfusions. ${ }^{48}$

A study by Pedrazzoli et al investigated the use of either an ESA plus intravenous iron or an ESA alone in patients with solid tumors undergoing chemotherapy, who were ironreplete but anemic (excluding patients with absolute or functional iron deficiency). There were statistically significant improvements in hemoglobin and hematopoietic responses in the intravenous iron group. ${ }^{49}$ These outcomes echo those of Henry et al, in which the response rate was $73 \%$ for patients receiving an ESA plus intravenous iron compared with $41 \%$ for an ESA alone. ${ }^{47}$

Nonresponders in the Pedrazzoli study (after four weeks) were given double the dose of ESA. This resulted in a $68.2 \%$ response in those receiving intravenous iron compared with $32 \%$ in those not receiving intravenous iron $(P=0.019) .{ }^{49}$

In the US, the Food and Drug Administration limits the use of an ESA agent to promote erythropoiesis in oncology patients to a ceiling of $\geq 100 \mathrm{~g} / \mathrm{L}$ hemoglobin. ${ }^{50}$ This reflects concerns that hemoglobin levels above $100 \mathrm{~g} / \mathrm{L}$ may adversely impact cancer progression. ${ }^{51}$ There is evidence from the renal arena to suggest that cancer progression may be caused by a direct effect of ESAs on the tumor and not the hemoglobin level. ${ }^{52}$ It is interesting to reflect on the focus of use of ESAs in the treatment of anemia associated with cancer without a parallel interest in the requirement for iron adequacy as a substrate for erythropoiesis.

In anemia associated with chronic kidney disease, both guidelines and clinical practice highlight the importance of iron adequacy when administering ESAs to prevent absolute or functional deficiency. The knowledge gained in the chronic kidney disease arena has not been heeded in the oncology arena, although several studies have concluded that the use of intravenous iron, as a supplement to ESAs, improves the efficacy of ESAs and can result in reduced dose requirements. ${ }^{45}$

The significance of intravenous iron, recognition of the possible coexistence of anemia of chronic disease, and the potential implications of iron deficiency and iron deficiency anemia in oncology patients, is now reflected in revisions of guidelines for the treatment of anemia associated with cancer. UK National Institute for Health and Clinical Excellence guidelines state that "erythropoietin analogues are recommended in combination with intravenous iron". ${ }^{53}$ EORTC guidelines also indicate that whilst there is no evidence that oral iron supplements increase the response to ESAs, there is evidence of a better response to ESAs with intravenous iron. The guidelines recommend use in absolute and functional iron deficiency. ${ }^{54}$ The American Society of Hematology and American Society of Clinical Oncology recommend monitoring iron parameters and correcting suboptimal levels in patients who are receiving ESA therapy. ${ }^{55}$

An insight into the required levels of intravenous iron repletion can be taken from the review of studies evaluating intravenous iron and ESAs in oncology. ${ }^{44}$ Doses are commonly up to $1000 \mathrm{mg}$ or higher when administered as a total dose infusion and are calculated using the Ganzoni formula. ${ }^{20,44,47}$

\section{Discussion}

Iron deficiency anemia and its precursor, iron deficiency, are associated with a number of clinical conditions. In many of these conditions, a blood transfusion has been the traditional approach to addressing iron deficiency anemia or iron deficiency, especially if oral iron has failed, is inappropriate, or a rapid resolution is required.

The development and introduction of safer intravenous iron preparations that offer the option of more flexible dosing 
has led to a re-evaluation of the role of intravenous iron, and provided clinicians with the facility to explore and develop the clinical use of these products. There is now a substantial evidence base of clinical papers that support the safety and efficacy of intravenous iron in a broad spectrum of clinical situations associated with iron deficiency and iron deficiency anemia. Studies comparing intravenous iron with oral iron, in addition to demonstrating equal or superior efficacy, often reflect on the more rapid repletion of iron stores and quicker elevation of hemoglobin levels. ${ }^{23,29,40,48}$ Furthermore, they highlight inadequacies of oral iron (side effects, poor compliance, poor absorption) and, in patients with continual blood loss, the inability to keep pace with the loss and sustain iron adequacy. ${ }^{23,29,40} \mathrm{~A}$ common requirement across the range of clinical situations is the need for higher and less frequent doses to achieve optimal clinical outcomes. The drivers of this practice also include overall cost reduction, improved patient convenience, improved compliance, preservation of venous access, and reduced blood transfusions (See Table 1). ${ }^{13,14,44,48}$

The introduction of ferric carboxymaltose and iron isomaltoside 1000, which do not require a test dose, and can be administered more rapidly and in higher doses, add a further dimension to the clinical proposition of intravenous iron. Furthermore, they distance intravenous iron further from oral iron as a source of iron repletion, especially as it is recognized that intravenous iron, and not oral iron, can overcome anemia of chronic disease. ${ }^{15}$

Much of the accumulated knowledge gained from the extensive use of intravenous iron in treating iron deficiency

Table I Summary of potential benefits of high-dose administrations of intravenous iron

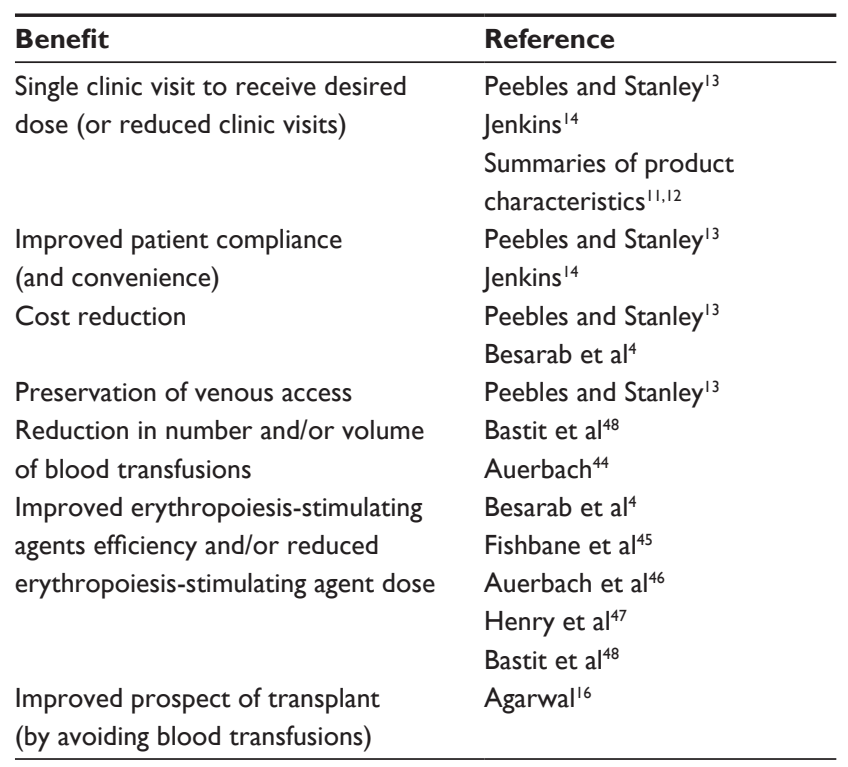

anemia and iron deficiency associated with chronic kidney disease has only slowly migrated to other disciplines. Thus, while blood transfusions for chronic kidney disease patients have virtually been eliminated, this is not the case in other clinical situations. The vast majority of chronic kidney disease patients progress to receive intravenous iron plus an ESA. Absolute and functional iron deficiency are well understood by renal clinicians and, because of this, guidelines recommend that iron adequacy be achieved prior to commencing an ESA. In low clearance (nondialysis-dependent) and chronic ambulatory peritoneal dialysis patients, and when undertaking iron repletion ahead of initiating ESA treatment, high-dose supplementation is common practice. Indeed, in renal medicine, high-dose intravenous iron alone can delay the need for ESA treatment in the low clearance (nondialysisdependent) chronic kidney disease population. ${ }^{13,19}$

Iron repletion prior to commencing an ESA will improve the efficacy of treatment, often resulting in lower doses, with accruing cost savings. This practice has been slow to transfer into other arenas where ESAs have been used, often in much higher dosage regimens, especially in oncology. As Auerbach indicates, in oncology patients blood transfusion rates have declined by only $50 \%$, whereas in patients with chronic kidney disease the elimination of blood transfusions has been almost total. ${ }^{44}$ The role of intravenous iron and the need to establish adequate iron repletion prior to commencing an ESA is now explicit in the various guidelines for diseases where iron deficiency and iron deficiency anemia are implicated. ${ }^{47,49,53-55}$

The recognition that anemia of chronic disease can be overcome using intravenous iron, and a greater understanding of the mechanism behind anemia of chronic disease have also encouraged wider intravenous iron use at higher dose levels. This may not only account for the success in treating anemia associated with chronic kidney disease but also inflammatory bowel disease (with or without an ESA) and again may suggest that intravenous iron can have a more profound role in patients being treated for cancer. Studies reported by Henry et al and Pedrazzoli et al support this role. ${ }^{47,49}$

Blood loss is apparent in several situations where highdose intravenous iron is used. In such situations, robust iron stores are needed to sustain erythropoiesis (with or without concurrent use of an ESA). This is particularly important in inflammatory bowel disease and menorrhagia where intravenous iron can sustain iron adequacy and keep pace with blood loss. This can avoid or reduce the need for blood transfusions. Indeed, prior to surgery, high doses of intravenous iron are being used in anticipation of blood loss to reduce the transfusion requirement or postsurgically to 
restore hemoglobin levels. The evidence base and guidelines for the role of high-dose intravenous iron are still emerging, hence their omission in this review.

The introduction of ferric carboxymaltose and, subsequently, iron isomaltoside 1000 are welcome developments. These provide intravenous iron in a form that will make it easier for clinicians to use and therefore encourage wider intravenous iron use across the range of conditions where the use of high-dose intravenous iron achieves good clinical outcomes.

The required dose of iron can be calculated using the Ganzoni formula, which is applicable across the spectrum of indications. This takes into account body weight, target hemoglobin level, and desired iron stores.

The introduction of ferric carboxymaltose has progressed intravenous iron repletion by offering high-dose treatment rapidly without a test dose. The subsequent introduction of iron isomaltoside 1000 may be considered to add to this profile by offering the prospect of administering even higher doses of intravenous iron in a single administration without compromising safety.

In addition to being able to deliver more iron to a patient in a single infusion, the use of iron isomaltoside 1000 is not constrained by the aluminum or sodium content in its formulation. Ferric carboxymaltose contains aluminum (up to $75 \mu \mathrm{g} / \mathrm{mL}$ ) and up to $5.5 \mathrm{mg}$ of sodium per $\mathrm{mL}$ which may be of concern in dialysis patients and those on sodium-restricted diets. ${ }^{11}$ The frequency of administration is limited to one infusion every seven days for both products.
The implications of the maximum iron dose based on body weight can be considered by examining doses that can be administered based on mean population adult body weights by gender in Europe and the US (See Tables 2 and 3).

From these tables it can be seen that, from body weight alone, the scope for administering high-dose infusions (above $1000 \mathrm{mg}$ iron) in a single infusion is higher with iron isomaltoside 1000 than with ferric carboxymaltose. The tables reveal that only women in Europe with a mean body weight of $65.8 \mathrm{~kg}$ can receive the maximum permitted $15 \mathrm{mg} / \mathrm{kg}$ iron dose of ferric carboxymaltose in a single infusion. Patients in the weight range $50-67 \mathrm{~kg}$, approximately $33 \%$ of the population, may receive a dose of $1000 \mathrm{mg}$ (or more) when iron isomaltoside 1000 is administered; however, only those weighing more than $67 \mathrm{~kg}$ can receive the maximum $1000 \mathrm{mg}$ dose of ferric carboxymaltose. ${ }^{11,12,56}$ The scope for giving higher doses of isomaltoside 1000 is far wider, suggesting that its use may avoid the need for two or more infusions and two or more visits to a hospital to receive the required calculated dose.

The relevance of these dose constraints should be considered in the context of the spectrum of dose levels detailed in the clinical conditions outlined. The need to administer high doses of intravenous iron to achieve desired target hemoglobin and ferritin levels is illustrated in the various potential clinical situations. In various studies, high doses are cited with ranges up to $1500 \mathrm{mg}$ of iron for nondialysis-dependent chronic kidney disease, $3600 \mathrm{mg}$ in inflammatory bowel disease, $1600 \mathrm{mg}$ in obstetrics, $2000 \mathrm{mg}$ in menorrhagia, and $3000 \mathrm{mg}$ in cancer (See Table 4). ${ }^{21,23,29,40,46}$

Table 2 Maximum permitted iron dose based on mean body weight: European population

\begin{tabular}{|c|c|c|c|c|c|}
\hline \multicolumn{3}{|c|}{ Male, mean adult weight 78.9 kg } & \multicolumn{3}{|c|}{ Female, mean adult weight $65.8 \mathrm{~kg}$} \\
\hline & $\begin{array}{l}\text { Maximum dose } \\
\text { based on body } \\
\text { weight }\end{array}$ & $\begin{array}{l}\text { Maximum dose } \\
\text { allowed according to } \\
\text { SPC } \\
11,12\end{array}$ & & $\begin{array}{l}\text { Maximum dose } \\
\text { based on body } \\
\text { weight }\end{array}$ & $\begin{array}{l}\text { Maximum dose } \\
\text { allowed according to } \\
\text { SPC }\end{array}$ \\
\hline Iron isomaltoside 1000 & 1578 mg* & $1578 \mathrm{mg}$ & Iron isomaltoside 1000 & $1316 \mathrm{mg} *$ & $1316 \mathrm{mg}$ \\
\hline Ferric carboxymaltose & $\mathrm{I} 183 \mathrm{mg}^{\dagger}$ & 1000 mg & Ferric carboxymaltose & $987 \mathrm{mg}^{\dagger}$ & 987 mg \\
\hline
\end{tabular}

Notes: tbased on $15 \mathrm{mg} / \mathrm{kg}$ body weight; *based on $20 \mathrm{mg} / \mathrm{kg}$ body weight. Source of population data. ${ }^{56}$

Abbreviation: SPC, summary of product characteristics.

Table 3 Maximum permitted iron dose based on mean body weight: US population

\begin{tabular}{|c|c|c|c|c|c|}
\hline \multicolumn{3}{|c|}{ Male, mean adult weight, $86.8 \mathrm{~kg}$} & \multicolumn{3}{|c|}{ Female, mean adult weight, 74.7 kg } \\
\hline & $\begin{array}{l}\text { Maximum dose } \\
\text { based on body } \\
\text { weight }\end{array}$ & $\begin{array}{l}\text { Maximum dose } \\
\text { allowed according to } \\
\text { SPC } \\
\text { 11,12 }\end{array}$ & & $\begin{array}{l}\text { Maximum dose } \\
\text { based on body } \\
\text { weight }\end{array}$ & $\begin{array}{l}\text { Maximum dose } \\
\text { allowed according to } \\
\text { SPC }{ }^{11,12}\end{array}$ \\
\hline Iron isomaltoside 1000 & 1736 mg* & $1736 \mathrm{mg}$ & Iron isomaltoside 1000 & 1494 mg* & 1494 mg \\
\hline Ferric carboxymaltose & $1302 \mathrm{mg}^{\dagger}$ & $1000 \mathrm{mg}$ & Ferric carboxymaltose & $1121 \mathrm{mg}^{\dagger}$ & $1000 \mathrm{mg}$ \\
\hline
\end{tabular}

Notes: 'Based on $15 \mathrm{mg} / \mathrm{kg}$ body weight; *based on $20 \mathrm{mg} / \mathrm{kg}$ body weight; Source of population data. ${ }^{57}$

Abbreviation: SPC, summary of product characteristics. 
Table 4 Summary of comparative profiles of iron isomaltoside 1000 and ferric carboxymaltose ${ }^{1,12,58,59}$

\begin{tabular}{lll}
\hline & Ferric carboxymaltose & Iron isomaltoside I000 \\
\hline Maximum dose per kg body weight & $15 \mathrm{mg} \mathrm{iron} / \mathrm{kg}$ body weight & $20 \mathrm{mg}$ iron//kg body weight \\
Maximum dose in a single administration & $1000 \mathrm{mg}$ & $20 \mathrm{mg}$ iron/kg body weight \\
Minimum body weight for $1000 \mathrm{mg}$ dose & $67 \mathrm{~kg}$ & $50 \mathrm{~kg}$ \\
Speed of administration & $<500 \mathrm{mg}$ in 6 minutes & $0-5 \mathrm{mg} / \mathrm{kg} \mathrm{in} \mathrm{I5} \mathrm{minutes}$ \\
& $500-1000 \mathrm{mg}$ in I5 minutes & $6-10 \mathrm{mg} / \mathrm{kg} \mathrm{in} 30 \mathrm{minutes}$ \\
& & $1 \mathrm{I}-20 \mathrm{mg} / \mathrm{kg}$ in $60 \mathrm{minutes}$ \\
Test dose required & No & No \\
Short chain carbohydrate structure & No & Yes \\
& Branched & Unbranched linear \\
& polysaccharides & oligosaccharides \\
\hline
\end{tabular}

The need to administer doses of $1000 \mathrm{mg}$ or more is not uncommon. In studies published on the use of ferric carboxymaltose, the need for more than a single infusion to achieve the required calculated dose to be administered is explicit. In inflammatory bowel disease, the authors indicate that ferric carboxymaltose was administered at " 1 -week intervals until the patients' calculated total iron deficit was reached". ${ }^{60}$ In a study reporting on the safety and tolerability of ferric carboxymaltose across a range of indications, 94\% of patients received a total calculated dose of $1000 \mathrm{mg}$ or more. ${ }^{32}$ In a study investigating the efficacy of ferric carboxymaltose in the treatment of heavy uterine bleeding, the authors indicate that five patients received a single infusion, 197 patients received two infusions, and 28 received three infusions $(\mathrm{n}=230)$. The mean dose was $1568 \mathrm{mg}$, and $97.8 \%$ received total doses that exceeded $1000 \mathrm{mg}$, the equivalent of iron in 5 units of blood. ${ }^{40}$

The need to be able to deliver high doses of iron in a number of clinical circumstances is unequivocal. Reducing the frequency of administrations will improve compliance and the burden of hospital visits to receive iron administrations. ${ }^{14}$ This is likely to be more convenient for both patients and health care professionals. Financial savings should accrue from a reduction in visits, reduced travel and transportation costs, use of giving sets, nursing time (preparation, setting up, and monitoring infusion delivery), and pharmacy preparation time. Further savings should accrue directly by avoiding functional iron deficiency when ESAs are being administered, thereby optimizing ESA use, and in some circumstances allowing a reduction in ESA dose levels. ${ }^{4,45}$

In this review we have not considered the relative adverse event profiles of the two preparations. A recent study in the US suggests that serious adverse events associated with intravenous iron therapy are rare. ${ }^{61,62}$

Whilst there is a difference in carbohydrate composition and molecular structure between iron isomaltoside 1000 and ferric carboxymaltose, the summaries of product characteristics suggest that the type and severity of any adverse events are likely to be similar. ${ }^{11,12}$ Real world data reporting of actual adverse events as the products are adopted in practice and future studies may reveal differences.

\section{Conclusion}

A review of clinical situations in which intravenous iron administration is appropriate reveals that high doses of iron supplementation are commonly required to optimize the management of iron deficiency and iron deficiency anemia either alone or concomitantly with an ESA. The benefits of intravenous compared with oral iron repletion are highlighted, especially in circumstances where there is chronic ongoing blood loss and where anemia of chronic disease is implicated.

The administration of high doses of iron can reduce the need for frequent hospital visits. This is more convenient for both patients and health care professionals. There are a number of cost savings associated with reduced frequency of administrations, including hospital visits to receive iron infusions. The number of infusions required to deliver the desired dose requirements may affect compliance, and therefore likelihood of achieving the required repletion and the speed of repletion. In many circumstances, the use of these products will directly result in a reduced demand for blood transfusions. This will help to preserve blood supplies and lessen the exposure to the risks associated with blood transfusions. Such accumulated savings may fully, or partially, offset the cost of these new medications.

The introduction of new intravenous iron formulations, especially in quick succession, suggests that existing options for treatment were not optimal. There has now been major progress in addressing these shortcomings because the introduction of ferumoxytol and ferric carboxymaltose realized the aim of not requiring a test dose whilst offering the 
ability to administer far higher single doses of iron rapidly. The ceiling imposed on the size of a single dose of ferric carboxymaltose $(15 \mathrm{mg} / \mathrm{kg}$ body weight up to a ceiling of $1000 \mathrm{mg}$ ) has been surpassed by the introduction of iron isomaltoside 1000 where the scope for a single infusion has been increased to $20 \mathrm{mg} / \mathrm{kg}$ body weight, without a ceiling, within an hour and without a test dose.

Recognition of the prevalence of iron deficiency and iron deficiency anemia and its association with a wide range of clinical situations, the understanding of the mechanism of anemia of chronic disease, and how this may be successfully addressed with intravenous iron, and the emerging prospect of iron being used to avoid or reduce the demand for blood transfusions, highlight the growing need for intravenous iron in higher single doses. Treatment options for iron deficiency anemia have taken a major leap forward in the past two years.

\section{Disclosure}

The author reports no conflict of interest in this work.

\section{References}

1. Nemeth E, Ganz T. The role of hepcidin in iron metabolism. Acta Hematol. 2009;122(2-3):78-86.

2. Craig R, Mindell J, editors. Health Survey for England 2005: The Health of Older People. Volume 1. London: TSO; 2007.

3. De Benoist B, McLean E, Egli I, Cogswell M, editors. Worldwide Prevalence of Anemia 1993-2005: WHO Global Database on Anemia. Geneva: World Health Organisation; 2005.

4. Besarab A, Amin N, Ahsan M, et al. Optimization of epoetin therapy with intravenous iron therapy in hemodialysis patients. $J$ Am Soc Nephrol. 2000;11:530-538.

5. Melamed N, Ben-Haroush A, Kaplan B, Yogev Y. Iron supplementation in pregnancy - does the preparation matter? Arch Gynecol Obstet 2007;276(6):601-604.

6. Lawrence R. Development and comparison of iron dextran products. PDA J Pharm Sci Technol. 1998;52(5):190-197.

7. Venofer ${ }^{\mathbb{8}}$ [Package insert]. Surrey, UK: Syner-med Pharmaceutical Products Ltd; 2010.

8. Cosmofer ${ }^{\circledR}$ [Package insert]. Holbaek, Denmark: Pharmacosmos A/S; 2010.

9. Ferrlecit ${ }^{\mathbb{}}$ [Monograph]. Bridgewater, NJ: Sanofi-aventis US LLC; 2010.

10. Feraheme ${ }^{\circledR}$ [Monograph]. Cambridge, MA: AMAG Pharmaceuticals Inc; 2009.

11. Ferinject ${ }^{\mathbb{}}$ [Package insert]. Surrey, UK: Syner-med Pharmaceutical Products Ltd; 2010.

12. Monofer ${ }^{\mathbb{B}}$ [Package insert]. Cambridge, MA: AMAG Pharmaceuticals Inc; 2010.

13. Peebles G, Stanley S. Evaluation of service reconfiguration for managing intravenous iron supplementation in non-hemodialysis patients with chronic renal failure. Journal of Outcomes Research. 2004;8: $15-25$.

14. Jenkins A. Using iron dextran to treat iron-deficiency anemia. Hospital Pharmacist. 2005;12(6):224-225.

15. Auerbach M, Coyne D, Ballard H. Intravenous iron: From anathema to standard of care. Am J Hematol. 2008;83(7):580-588.

16. Agarwal R. Individualizing decision-making - resurrecting the doctorpatient relationship in the anemia debate. Clin J Am Soc Nephrol. 2010 5(7):1340-1346.
17. The Renal Association. Clinical Practice Guidelines: Chronic Kidney Disease. Hampshire, UK: The Renal Association; 2010.

18. Jungers P, Qualim Z, Nguyen-Khoa T, Massey Z, London G. Cardioprotection: An essential component for predialysis chronic renal failure treatment. Nephrologie. 2003;24(2):79-88. French.

19. Macdougall IC. Iron supplementation in the non-dialysis chronic kidney disease (ND-CKD) patient: Oral or intravenous? Curr Med Res Opin. 2010;26(2):473-482.

20. Ganzoni AM. Schweiz Med Wochenschr. 1970;100(7):301-303. German. [Intraverous iron-deptran: therapeutic and experimental possibilities]. [German]

21. Peebles G, Fenwick S. Intravenous iron administration in a short-stay hospital setting. Nurs Stand. 2008;22(48):35-41.

22. Gasche C, Lomer MCE, Cavill, Weiss G. Iron, anemia, and inflammatory bowel disease. Gut. 2004;53:1190-1197.

23. Gasche C, Berstad A, Befrits R, et al. Guidelines on the diagnosis and management of iron deficiency and anemia in inflammatory bowel disease. Inflamm Bowel Dis. 2007;13(12):1545-1553.

24. Bothwell TH. Iron requirements in pregnancy and strategies to meet them. Am J Clin Nutr. 2000;72(1):S257-S264.

25. National Institute for Health and Clinical Excellence. Antenatal care: Routine Care for the Healthy Pregnant Woman. Clinical Guideline. London, UK: National Institute for Health and Clinical Excellence; 2008.

26. Bashiri A, Smolin A, Sheiner E, Zelingher J, Mazor M. Maternal rehospitalization after singleton term vaginal delivery. J Matern Fetal Neonatal Med. 2003;14(5):344-348.

27. Knight M, Callaghan WM, Berg C, et al. Trends in postpartum hemorrhage in high resource countries: A review and recommendations from the International Postpartum Hemorrhage Collaborative Group. BMC Pregnancy Childbirth. 2009;9:55.

28. Royal College of Obstetricians and Gynecologists. National Sentinel Caesarean Section Audit (England, Wales and Northern Ireland). London, UK: Royal College of Obstetricians and Gynecologists Press; 2001.

29. Breymann C. The use of iron sucrose complex for anemia in pregnancy and the postpartum period. Semin Hematol. 2006;43 Suppl 6: S28-S31.

30. Bodnar LM, Siega-Riz AM, Miller WC, Cogswell ME, McDonald T. Who should be screened for postpartum anemia? An evaluation of current recommendations. Am J Epidemiol. 2002;156(10): 903-912.

31. Hallak M, Sharon AS, Diukman R, Auslender R, Abramovici H Supplementing iron intravenously in pregnancy. A way to avoid blood transfusions. J Reprod Med. 1997;42(2):99-103.

32. Qunibi W, Dinh Q, Benjamin J. Safety and tolerability profile of ferric carboxymaltose (FCM) a new high dose intravenous iron, across ten multi-center trials. J Am Soc Nephrol. 2007;18:SU-PO1029.

33. Protheroe J. Modern management of menorrhagia. J Fam Plann Reprod Health Care. 2004;30(2):118-122.

34. Oehler MK, Rees MC. Menorrhagia: An update. Acta Obstet Gynecol Scand. 2003;82(5):405-422.

35. Morrison J, Patel ST, Watson W, Zaidi QR, Mangione A, Goss TF. Assessment of the prevalence and impact of anemia on women hospitalized for gynecologic conditions associated with heavy uterine bleeding. J Reprod Med. 2008;53(5):323-330

36. Lo L. Modern management of menorrhagia. Hong Kong Practitioner. 1996;18(2):62-66.

37. Prentice A. Medical management of menorrhagia. BMJ. 1999;319: 1343-1345.

38. National Institute for Health and Clinical Excellence. Heavy Menstrual Bleeding. Clinical Guideline. London, UK: National Institute for Health and Clinical Excellence; 2007.

39. Kim YH, Chung HH, Kang SB, Kim SC, Kim YT. Safety and usefulness of intravenous iron sucrose in the management of preoperative anemia in patients with menorrhagia: A phase IV, open label, prospective, randomized study. Acta Haematol. 2009;121(1):37-41. 
40. Van Wyck DB, Mangione A, Morrison J, Hadley PE, Jehle JA, Goodnough LT. Large-dose intravenous ferric carboxymaltose injection for iron deficiency anemia in heavy uterine bleeding: A randomized, controlled trial. Transfusion. 2009;49(12):2719-2728.

41. Bokemeyer C, Aapro MS, Courdi A, et al. European Organisation for Research and Treatment of Cancer (EORTC) Taskforce for the Elderly. EORTC guidelines for the use of erythropoietic proteins in anemic patients with cancer. Eur J Cancer. 2004;40(15):2201-2216.

42. Crawford J, Cella D, Cleeland CS, et al. Relationship between changes in hemoglobin level and quality of life during chemotherapy in anemic cancer patients receiving epoetin-alfa therapy. Cancer. 2002;95(4): 888-895.

43. Weiss G. Pathogenesis and treatment of anemia of chronic disease. Blood Rev. 2002;16(2):87-96.

44. Auerbach M. Should intravenous iron be the standard of care in oncology. J Clin Oncol. 2008;26(10):1579-1581.

45. Fishbane S, Frei GL, Maesaka J. Reduction in recombinant human erythropoietin doses by the use of chronic intravenous iron supplementation. Am J Kidney Dis. 1995;26(1):41-46.

46. Auerbach M, Ballard H, Trout JR, et al. Intravenous iron optimizes the response to recombinant human erythropoietin in cancer patients with chemotherapy related anemia: A multicenter, open label, randomized trial. J Clin Oncol. 2004;22(7):1301-1307.

47. Henry DH, Dahl NV, Auerbach M, Tchekmedyian S, Laufman LR. Intravenous ferric gluconate improves response to epoietin alfa versus oral iron or no iron in anemic patients with cancer receiving chemotherapy. Oncologist. 2007;12(2):231-242.

48. Bastit L, Vandebroek A, Altintas S, et al. Randomized, multicenter, controlled trial comparing the efficacy and safety of darbepoietin alpha administered every 3 weeks with or without intravenous iron in patients with chemotherapy-induced anemia. J Clin Oncol. 2008;26(10): 1611-1618

49. Pedrazzoli P, Farris A, Del Prete S, et al. Randomized trial of intravenous iron supplementation in patients with chemotherapy-related anemia without iron deficiency treated with darbepoietin alfa. J Clin Oncol. 2008;26(10):1619-1625.

50. American Society of Clinical Oncology. Important information for physicians about changes affecting the FDA-approved use of erythropoiesis stimulating agents (ESA). Available from: http:// www.asco.org/ASCO/ArticleASCO/Cancer\%20Policy\%20and\%20 Clinical\%20Affairs/Policy/MMA\%20Regulation\%20\&\%20Resources/ erythropoieticstimulatingagents $\% 20$ Doc $\% 20$ for $\% 20$ Practicing $\% 20$ Oncologists\%20092508\%20FINAL.pdf. Accessed Jun 82010.
51. Steensma DP. Is anemia of cancer different from chemotherapy induced anemia? J Clin Oncol. 2008;26(7):1022-1024.

52. Singh AK, Tang KL, Bamhart $\mathrm{H}$, et al. CHOIR investigators. Correction of anemia with epoietin alfa in chronic kidney disease. $N$ Engl $J$ Med. 2006;355(20):2085-2098.

53. National Institute for Health and Clinical Excellence. Erythropoietin analogues for anemia caused by cancer treatment. Technology appraisal guidance. London, UK: National Institute for Health and Clinical Excellence; 2008.

54. Aapro MS, Link H. September 2007 Update on EORTC guidelines and anemia management with erythropoiesis-stimulating agents. Oncologist. 2008;13 Suppl 3:33-36.

55. Steensma DP. New ASH/ASCO guidelines on the use of erythropoiesisstimulating agents: A chorale amid cacophony. J Support Oncol. 2007; 5(10):471-473

56. Special Eurobarometer. Health and Food. 246/Wave 64.3 - TNS Opinion and Social. European Commission; 2006.

57. Portier K, Tolson JK, Roberts SM. Body weight distributions for risk management. (NHANES 1999-2002). Risk Anal. 2007;27(1):11-26.

58. European Pharmacopoeia - Monograph. Page 1408.

59. Luitpold Pharmaceuticals Inc. (19th July 2007). Methods and Compositions for Administration of Iron. WO2007/081744 A2.

60. Kulnigg S, Stoinov S, Simanenkov V, et al. A novel intravenous iron formulation for treatment of anemia in inflammatory bowel disease: The ferric carboxymaltose (FERINJECT) randomized controlled trial. Am J Gastroenterol. 2008;103(5):1182-1192.

61. Wysowski DK, Swartz L, Borders-Hemphill BV, et al. Use of parenteral iron products and serious anaphylactic-type reactions. Am J Hematol. 2010;85(9):650-654.

62. Chertow GM, Winkelmayer WC. On the relative safety of intravenous iron formulations: New answers, new questions. Am J Hematol. 2010; 85(9):643-644.
Drug Design, Development and Therapy

\section{Publish your work in this journal}

Drug Design, Development and Therapy is an international, peerreviewed open-access journal that spans the spectrum of drug design and development through to clinical applications. Clinical outcomes, patient safety, and programs for the development and effective, safe, and sustained use of medicines are a feature of the journal, which

\section{Dovepress}

has also been accepted for indexing on PubMed Central. The manuscript management system is completely online and includes a very quick and fair peer-review system, which is all easy to use. Visit http://www.dovepress.com/testimonials.php to read real quotes from published authors.

Submit your manuscript here: http://www.dovepress.com/drug-design-development-and-therapy-journal 